\title{
Effect of 8-Week Circuit Weight Training on Strength
}

\author{
Hartati Hartati*, Wahyu Indra Bayu, Silvi Aryanti \\ Physical Education and Health, Faculty of Teacher Training and Education \\ Sriwijaya University \\ South Sumatera, Indonesia \\ *hartati@fkip.unsri.ac.id, wahyu.indra@fkip.unsri.ac.id, silviaryanti@fkip.unsri.ac.id
}

\begin{abstract}
The aim of this study was to know the effect of weight training to enhance service capabilities. Subject was Volleyball Extracurricular at Senior High School 1 Indralaya Palembang, and collected using total sampling $(\mathrm{N}=30)$. Through a quasi-experimental treatment given to the subject. Subjects completed in a randomized order three circuit weight training protocols of the same duration ( 3 sets of 8 exercises, $45 \mathrm{~min}$ ) and intensity (70\% of 1 repetition maximum). The results showed that there was difference between pre-test and post-test data (p-value $>0,05$ ). It can be concluded that the circuit weight training can be used to improve strength in volleyball player. The implication of this research is that weight training by using circuit method can be used as one type of exercise to improve the strength of volleyball athletes.
\end{abstract}

Keywords: circuit training, strength, weight training

\section{INTRODUCTION}

One important factor for training in sports is strength, as is volleyball. One factor in every basic technique of volleyball is strength. Strength and muscle power of both upper and lower extremities as well as neuromuscular coordination and technical skills are elements that a good volleyball player aims to develop [1]. Several studies have investigated the anthropometric and physiological characteristics of both teenage and adult volleyball players and their impact on volleyball performance [1-5]. Passing in spite of accuracy, it also requires strength, Set-up requires strength to push the ball up in the pass. It is also very important in the smash and blocking technique. While in the serve technique, hand strength is needed to send the ball through the net to the opponent's area. For this reason, it is necessary train the strength of the volleyball player's hand muscles in order to play volleyball properly. Weight training is one form of training that is needed. Weight training is training that is carried out systematically by using weights to increase muscle strength and function to improve physical condition, prevent injury or for health purposes. Weight training can be done by using weights from one's own weight (internal weights) or using external weights as it called free weights such as dumbbells, barbells, or gym machines. Forms of training that use the most widely used weights are chin-ups, push-ups, sit-ups, or back-ups, while using outside weights varies according to the purpose of the exercise.
Weight training is a type of resistance training to increase the strength and size of skeletal muscles primarily using bars, dumbbells and/or other equipment. Weight training is necessary for sports like bodybuilding, weightlifting and powerlifting where strength, power, and/or muscle mass are necessary. It is used in many other sports, such as football, wrestling, and rowing, in order to increase the performance of athletes and reduce the frequency and severity of injuries [6]. Increased strength also improves the capacity to perform everyday tasks more easily. Weight training is further associated with reductions in anxiety symptoms, improvements in sleep quality and improvements in self-esteem [7]. Studies with trained men showed that "muscle group training frequency" of 2 or 3 times per week yielded better strength results compared to once a week, with no apparent further advantage of three versus two sessions [8]. A metaanalysis focusing on strength improvements found that for untrained and trained individuals, frequencies of 3 and 2 times per week respectively gave the best (strength) results $[9,10]$. However, in each of the above reviews, the total weekly volumes (sets $x$ repetitions $\times$ resistance) between the groups were not matched.

More efficient programs can be designed for athletes who possess physiological capabilities. Therefore, utilizing some appropriate strategies by the experts and trainers will bring desirable consequences. Similarly, the time and the cost spent for the preparation of each athlete will be decreased. Since weight training at this age necessitates special care and attention, and there are very few researches in this regard, this paper examines the effect of weight training to increase of strength.

\section{METHOD}

This research is a quasi-experimental research with one group pre-test and post-test design. 30 samples, age \pm 17 , do circuit weight training during 8 weeks and the frequency of training 4 times a week. All participants were randomly recruited from volleyball extracurricular at Senior High School 1 Indralaya Palembang. Participants and their parents were informed on the purposes of the study, the methods and the possible risks involved and informed consents were signed by the children's parents. Subjects completed in a randomized order three circuit weight training protocols of the same duration ( 3 sets of 10 exercises, $45 \mathrm{~min}$ ) and intensity (70\% of 1 repetition maximum). The circuit protocols were composed of 
free weight exercises. The Circuit Weight Training protocol was performed as follows: push up, proper push up, seated dip triceps, pull up, bird dog, straight arm plank ball, straight arm plank - single-leg hip extension (feet elevated), straight-arm plank - suspended upper (single-leg hip extension), box walk up, suspended lower (walk out and back). To analyse differences strength data before and after the treatment, we used paired sample t-test when the differences was significant $(\mathrm{p}<0,05)$ according to the result of paired sample t-test.

\section{RESULTS AND DISCUSSION}

Based on table 1 the mean value of the study sample was 20,73 (pre-test) and 25,70 (post-test), the standard deviation was 1,337 (pre-test) and 1,368 (post-test) and the increase was $9.178 \%$.

TABLE I. DATA DESCRIPTION

\begin{tabular}{|l|l|l|l|}
\hline & \multicolumn{1}{|c|}{ Pre-test } & \multicolumn{1}{|c|}{ Post-test } & Increase \\
\hline $\mathrm{N}$ & 30 & 30 & \multirow{3}{*}{$23,95 \%$} \\
\cline { 1 - 3 } Minimum & 17 & 22 & \\
\cline { 1 - 3 } Maximum & 23 & 28 & \\
\cline { 1 - 3 } Mean & 20.73 & 25.70 & \\
\cline { 1 - 3 } Std. Deviation & 1.337 & 1.368 & \\
\cline { 1 - 3 } & & &
\end{tabular}

Then the hypothesis will be tested to answer the research question. But previously the normality and homogeneity test of the data was carried out as a prerequisite for testing the hypothesis. Tests for normality and homogeneity of data are shown in table 2 and table 3 below:

TABLE II. ONE-SAMPLE KOLMOGOROV-SMIRNOV TEST

\begin{tabular}{|l|l|l|}
\hline \multicolumn{1}{|c|}{ Data } & \multicolumn{1}{|c|}{ Pre-test } & \multicolumn{1}{c|}{ Post-test } \\
\hline $\mathrm{N}$ & 30 & 30 \\
\hline Asymp. Sig. (2-tailed) & 0.053 & 0.069 \\
\hline
\end{tabular}

In table 2 it appears that the p-value is 0.053 for the pre-test and 0.069 data for the post-test data. Because the p-value > 0.005 , it can be concluded that the pre-test and post-test data are normally distributed.

TABLE III. TEST OF HOMOGENEITY OF VARIANCES

\begin{tabular}{|c|l|l|c|}
\hline Levene Statistic & \multicolumn{1}{c|}{ df1 } & df2 & Sig. \\
\hline 0.000 & 1 & 58 & 0.860 \\
\hline
\end{tabular}

In table 3 it appears that the p-value is 0.860 , because the $p$ value $>0.005$, it can be concluded that the pre-test and post-test data are homogeneous.

\section{TABLE IV. PAIRED SAMPLES TEST}

\begin{tabular}{|l|l|}
\hline$T$ & 149.000 \\
\hline Df & 29 \\
\hline Sig. (2-tailed) & 0.000 \\
\hline
\end{tabular}

In table 4, statistical differences for the data pre-test and post-test were analysed using paired samples t-test for the total score of the strength, the results showed significant difference in strength before and after treatment using the method of circuit weight training $[t=149,00 ; p=0.000(<0.05)]$.

Training is a type of physical activity carried out in a structured planning, repetitive body movements aimed at increasing or maintaining one or more components of physical fitness $[11,12]$. The basic principle of training is an effort to improve skills and achievements while the good training principle is to produce a good training organization. This weight training is done by paying attention to the principles of training, namely the individual principle, the principle of variation, the principle of the training model, and the principle of increasing load. Circuit weight training during 8 weeks and the frequency of training four times a week. This is supported by Mylsidayu and Moraes that an increase in weight training occurs within 2-6 weeks with the DNLP linear periodization program with 20 reps and $50 \%$ of weight using bench press, leg press, front lat pull-down, leg extensions, military press, seated leg curls, pulley triceps extensions, abdominal crunches, and arm curls significantly increased bench press 15RM (175 and $219 \%$ ), leg press 15RM (395 and 455\%), maximal oxygen consumption (VO2 max) (9 and 10\%), and free fat mass (2.8 and 3\%) $[13,14]$. Whereas Park said that a combination of aerobic exercise in the form of running with $40 \%-75 \%$ MHR and weight training with $60 \%-70 \% 1$ RM [15], with 8-10 reps and 3 sets can significantly increase grip strength, push-ups, and intake maximum oxygen (VO2max) [16]. Showed that the application of specific strength training programs can improve the performance capabilities of volleyball player skills.

Systematic training resulted structural and functional changes, or adaptations, in the body. This level of adaptation is evidenced by the size and strength of the muscles. The magnitude of this adaptation is directly proportional to the demands placed on the body by volume, frequency, and intensity (load) of training, as well as the body's ability to adapt to these demands. Rational training adjusts to increased physical performance pressure. In other words, if the body is given a rational training load or in accordance with the body's physical needs and with sufficient recovery time given to the physiological system, the body will automatically adjust to the training and become stronger. Circuit training can be used by one individual or group and does not require complicated equipment. Circuit training involves a series of exercises with little or no rest at the end between each exercise. General circuit training consists of a number of different exercises, usually between 10 and 15 forms of exercise commonly called a post. At each post of the circuit training the individual must do about 15 repetitions in 40 seconds, while the intensity of resistance training should not exceed $60 \%$ of one maximum repetition, with short rest periods between exercises [17]. Training programs that are characterized by high volumes, have short rest periods and include some exercise and exercise, can be effectively used for body fat reduction [18]. Furthermore, [19], found that circuit training is an effective training method to increase endurance of local muscles, due to its very continuity and minimal rest between posts. 
[15] S.M. Park, Y.S Kwak and J.G Ji, "The effect combined exercise on health-related fitness, endotoxin, and immune function of postmenopausal woman with abdominal obesity," Journal of Immunology Research, pp. 830567-830575, 2015.

training on $70 \% 1 \mathrm{RM}$ can be helpful for improving in strength These are most important variables for volleyball skills. It seems that exercise trainings like those implicated in this study are safe and effective methods for improvement of young volleyball players' motor skills.

\section{CONCLUSION}

Based on the results and data analysis of the research, it concluded that there was an effect of weight training through the circuit method toward the strength. The implication of this research is that weight training by using circuit method can be used as one type of exercise to improve the strength of volleyball athletes.

\section{REFFERENCES}

[1] D.J. Smith, D. Roberts and B. Watson, "Physical, physiological and performance differences between Canadian national team and Universidad volleyball players," J Sports Sci, vol. 10, pp. 131-138, 1992.

[2] T. Gabbett and B. Georgieff, "Physiological characteristics of elite junior volleyball players over competitive season," J Strength Cond Coach, vol. 13, pp. 2-7, 2005.

[3] T. Gabbett and B. Georgieff, "Physiological and anthropometric characteristics of Australian junior national, state, and novice volleyball players," J Strength Cond Res, vol. 21, pp. 902-908, 2007.

[4] S.J. Fleck, S. Case, J. Puhl and P. Van Handle, "Physical and physiological characteristics of elite women volleyball players," Can J Appl Sport Sci, vol. 10, pp. 122-126, 1985.

[5] M. Thissen-Milder and J.L. Mayhew, "Selection and classification of high school volleyball players from performance tests," J Sports Med Phys Fitness, vol. 31, pp. 380-384, 1991.

[6] I. Shaw, B. Shaw, G. Brown and A. Shariat, "Review of the role of resistance training and musculoskeletal injury prevention and rehabilitation," Gavin Journal of Orthopedic Research and Therapy, pp. $1-5,2016$.

[7] P. O'Connor, M. Herring and A. Caravalho, "Mental health benefits of strength training in adults," American Journal of Lifestyle Medicine, vol. 4(5), pp. 377-396, 2010.

[8] M. Peterson, M. Rhea and B. Alvar, "Maximizing strength development in athletes: a metaanalysis to determine the dose-response relationship," The Journal of Strength and Conditioning Research, vol. 18(2), pp. 377 382, 2004

[9] M. Rhea, B. Alvar, L. Burkett and S. Ball, "A meta-analysis to determine the dose response for strength development," Medicine \& Science in Sports \& Exercise, vol. 35(3), pp. 456-464, 2003.

[10] A. Tzur and B. Roberts, Scientific recommendations for strength and hypertrophy training from 150+ studies (part 1 of 3), 2017, retrieved from: $\quad$ http://sci-fit.net/2017/scientificrecommendations1/?utm_campaign=Revue\%20newsletter\&utm_mediu $\mathrm{m}=$ Newsletter\&utm_source=revue\#Conclusion_for_frequency_strength and_hypertrophy.

[11] W.K. Hoeger and A. Hoeger, Lifetime physical fitness and wellness; a personalized program, 11th ed., Wadsworth, Cengage Learning, 2011.

[12] American College of Sports Medicine, ACSM'S Guidelines for Exercise Testing and Prescriptio, 9th ed., American College of Sports Medicine, 2014.

[13] A. Mylsidayu and F. Kurniawan, Ilmu Kepelatihan Dasar, Bandung: Alfabeta, 2015.

[14] E. Moraes, S.J. Fleck, D.M. Ricardo and R. Simão, "Effects on strength, power, and flexibility in adolescents of nonperiodized vs. daily nonlinear periodized weight training," Journal of Strength and Conditioning Research, vol. 27(12), pp. 3310-3321, 2013.

[16] S.H. Shakya, "The effect of specific weight training programme on the performance of skill ability of volleyball players," Indian Streams Research Journal, vol. 4(5), pp. 1-4, 2014.

[17] S. Romero-Arenas, M. Martínez-Pascual and P.E. Alcaraz, "Impact of resistance circuit training on neuromuscular, cardiorespiratory and body composition adaptations in the elderly," Aging and Disease, vol. 4(5), pp. 256-263, 2013.

[18] M.B. Miller, G.E.P. Pearcey, F. Cahill, H. McCarthy, S.B.D. Stratton, J.C. Noftall and D.C. Button, "The effect of a short-term high -intensity circuit training program on work capacity, body composition, and blood profiles in sedentary obese men: A pilot study," BioMed Research International, pp. 1-10, 2014.

[19] American College of Sports Medicine Position Stand, "Progression models in resistance training for healthy adults," Medicine \& Science in Sports \& Exercise, vol. 41(3), pp. 687-708, 2009. 